\title{
LISA in 2012 and Beyond: 20 Years After the First Proposal
}

\author{
Gerhard Heinzel and Karsten Danzmann
}

\begin{abstract}
After 20 years of study as a joint ESA-NASA mission, LISA had to be redesigned as an ESA-only mission in 2011/2012 to meet programmatic and budgetary constraints of the space agencies. The result is a mission concept called "eLISA" or "NGO" with two arms instead of three and one million km armlengths instead of 5, which results in smaller launch mass but still provides revolutionary science. Nevertheless, even the reduced science is expected to be revolutionary for the study of black holes and other astrophysical and cosmological questions. "eLISA"/"NGO" was not selected in ESA's call for the first ("L1") large mission in the Cosmic Vision program, but is a strong candidate for the L2 call, with possible international contributions from the US and/or China.
\end{abstract}

\section{Introduction}

Gravitational Waves have been predicted in 1916 by Albert Einstein as a consequence of GR. So far they have not yet been directly observed, despite the enormous efforts invested in resonant detectors and ground-based interferometers since the 1960s, culminating in the present LIGO, VIRGO, GEO600 and similar detectors. These are ultra-sensitive Michelson-type laser interferometers with km-scale armlengths and sophisticated vibration-isolation systems. Nevertheless, they are insensitive at frequencies below about $10 \mathrm{~Hz}$ because of inevitable disturbances on Earth such as seismic and gravity gradient noise. A first direct detection with these instruments is expected in a few years when the next generation with yet another huge step in sensitivity improvement goes online.

\footnotetext{
G. Heinzel $(\bowtie) \cdot$ K. Danzmann

Max-Planck-Institut für Gravitationsphysik (Albert-Einstein-Institut), Callinstrasse 38,

30167 Hannover, Germany

e-mail: gerhard.heinzel@aei.mpg.de

K. Danzmann

e-mail: karsten.danzmann@aei.mpg.de
} 
The majority of expected sources, however, have frequencies well below $10 \mathrm{~Hz}$ and are therefore only accessible from space. For their detection, space-based laser interferometers have been studied since the 1980s. LISA was selected as an ESA cornerstone mission in 1995, and the concept of a collaborative ESA/NASA mission with 3 spacecraft in heliocentric orbits trailing the Earth and inclined by $60^{\circ}$ against the ecliptic first appeared in 1997. This basic mission concept is basically unchanged until today.

The mission design was refined in great detail between 2004 and 2010 in an joint ESA/NASA Mission Formulation study that included an ESA-sponsored industrial study by EADS Astrium. Both the feasibility and scientific case were scrutinized in numerous reviews both in Europe and the US, such as the NRC's Beyond Einstein Program Assessment Committee (BEPAC) in 2006-7 and the Astro2010 Astronomy and Astrophysics Decadal Survey. All these reviews attested LISA a compelling and convincing science case as well as technical feasibility. Literally thousands of papers have been published on LISA sources, data analysis and instrumentation.

\section{Planned ESA Cosmic Vision L1 Selection in 2011}

In preparation for the call for proposals for the first "Large" mission (L1) in the ESA Cosmic Vision program, an Assessment Study Report ("Yellow Book") was prepared in February 2011 [1] that summarizes the scientific objectives, mission design and most important literature.

At that time three missions were in competition: LISA, the International X-ray Observatory (IXO), and the Europa Jupiter System Mission (EJSM-Laplace), all of which were conceived as ESA-NASA partnerships with about equal contributions.

In March 2011, however, ESA announced that due to budgetary constraints in NASA related to (among other reasons) the James Webb Space Telescope, none of these three missions could rely on the required NASA contribution [2]. Thus, the L1 downselection was postponed by one year, and the three missions were given the homework to modify their mission concepts such that they fit into an ESA-only envelope.

The LISA team studied different options, supported by industry and a mission concept study in ESA's concurrent design facility (CDF) in June/July 2011, and concluded that the "eLISA"/"NGO" concept described below would be the optimal mission within the given constraints. A new version of the "Yellow Book" was prepared and published in January 2012 [3].

\section{The eLISA/NGO Mission Concept}

Since the name LISA was considered to refer to the specific ESA/NASA mission described in [1], with 6 arms of 5 million km length etc., it was required to find a new name for the rescoped mission concept. The two names eLISA ("evolved LISA") 
and NGO ("New Gravitational wave Observatory") were chosen and used interchangeably for this purpose. See, however, Sect. 6 below regarding current naming conventions. The given constraints were a total cost for ESA of $850 \mathrm{M} €$, and member state contributions of about $200 \mathrm{M} €$. It quickly turned out that the most efficient way to reduce the predicted cost while maintaining as much of the science as possible is to reduce the launch mass and volume and the mission lifetime.

This was achieved by

- shrinking the telescope from $38 \mathrm{~cm}$ diameter to $20 \mathrm{~cm}$, with subsequent reductions also in length and height of the payload,

- shrinking the armlength from 5 to 1 million $\mathrm{km}$, which requires less fuel for planechange maneuvers to leave the ecliptic plane and helps to restore the reduced light power levels due to the smaller telescope,

- omitting the last maneuver of the cruise phase, resulting in a "drift-away" orbit with ever increasing distance to the Earth,

- omitting the third arm, resulting in a ' $\mathrm{V}$ ' shaped configuration instead of a full triangle.

Especially the omission of the third arm obviously leads to further cost reductions beyond the reduced launch mass, since less payload hardware is required. Other factors that contributed to the cost saving are:

- reducing the required mission lifetime from 5 to 2 years, with associated savings in on-ground testing and operations cost,

- providing the science instrument by an ESA memberstate consortium.

Apart from the above changes, the NGO payload is basically unchanged from LISA, and would be mounted into 3 identical spacecraft buses based on the LISA Pathfinder design, one 'mother' at the vertex of the ' $\mathrm{V}$ ' and two simpler 'daughter' spacecrafts at the ends. The two lighter daughter would together fit into one Soyuz launcher, while a second launcher is used for the mother spacecraft.

These reductions come, of course, at a cost in instrument performance. Figure 1 shows the strain sensitivity in comparison.

A significant qualitative reduction in science output results from the omission of the third arm: The NGO standard Michelson interferometer cannot instantaneously disentangle two possible mechanisms for a reduction in signal amplitude: larger distance to the source or polarization mismatch to the antenna orientation. The LISA triangle with 6 links, however, can simultaneously measure both polarizations and thereby provide absolute distance measurements to sources whose brightness is known, e.g., through the time evolution of the waveform. This shortcoming of NGO is somewhat mitigated, however, for sources that are observed over a significant fraction of a year, since the rotation of the constellation restores some of the polarization sensitivity. NGO would observe thousands of Compact White Dwarf Binaries (CWDB), hundreds of black hole binary inspirals and dozens of Extreme Mass Ratio Inspirals (EMRI). 

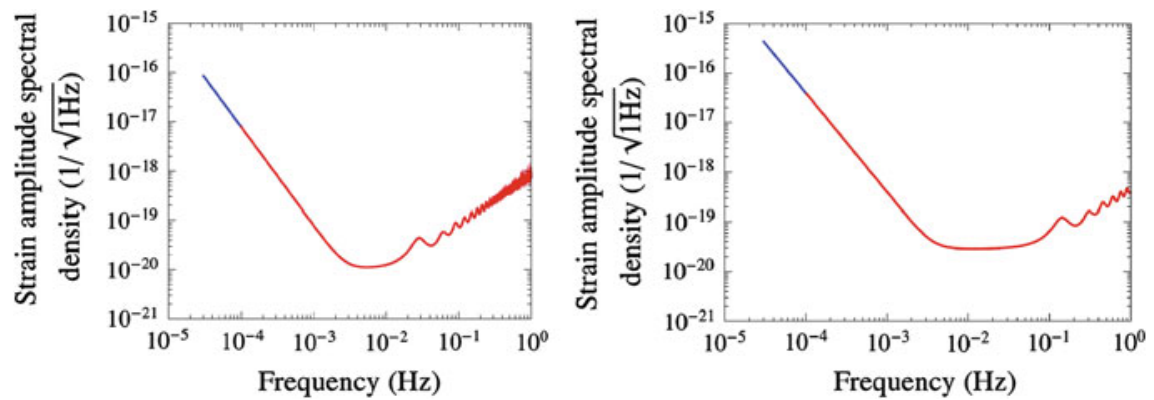

Fig. 1 Strain sensitivity of LISA (left) and NGO (right) in comparison (source [1, 3])

\section{The eLISA Consortium}

A significant programmatic change between LISA and NGO was the definition of an 'instrument' that would be delivered by the member states. That instrument consists of most of the scientific payload, with the exception of the lasers and telescopes. Hence, the instrument contains:

- the test masses in their Gravitational Reference Sensors, including front-end electronics and discharging equipment,

- the optical benches, including mechanisms and photoreceivers,

- the phasemeters and

- integration and testing tasks.

An important difference to the LISA planning is the phasemeter, which used to be a NASA contribution, but for eLISA/NGO needs to come from Europe. An ESA technology development contract granted to a Danish/German team will be completed in early 2013 and will produce a European phasemeter with all required functionality and performance. A consortium consisting of institutes and space agencies in Denmark, France, Germany, Italy, Spain, Switzerland and UK was formed and committed to develop and deliver the instrument.

\section{The ESA L1 Decision and Its Aftermath}

In early May 2012, the ESA Science Programme Committee (SPC) chose the Jupiter moon mission JUICE (evolved from EJSM-Laplace) as L1 mission to be launched in 2022. Reasons for not selecting eLISA/NGO in spite of its repeatedly praised scientific value and technological readiness, as judged by ESA itself, were not clear; possibly having to do with remaining concerns about the risk of such a revolutionary new instrument and the desire to wait for the results of LISA Pathfinder, now planned to be launched in 2015. At the same time ESA announced to continue the technology development for eLISA/NGO. 
The 9th LISA Symposium was held in Paris from May 21-25, 2012, with wide participation of scientists from Europe, the US and China. The community resolved to push forward with the LISA concept and submit an unbeatable entry for the L2 call for proposals in ESA's Cosmic Vision program, expected to occur in 2013 or 2014. The 'instrument' definition and consortium were maintained and are in function now. The first meeting of the new eLISA consortium took place October 22-23, 2012, also in Paris, and it was decided to form several working groups for science and technology. Both the consortium and ESA agree that third-party contributions of up to $20 \%$ of the mission cost are welcome, e.g. from the US or China, provided they are non-essential, i.e. European alternative suppliers are available in case of necessity.

\section{Naming the Mission Concepts}

At the 9th LISA Symposium in Paris it was decided to retain the name LISA for the general concept of a gravitational wave detector in space, consisting of a triangle in inclined heliocentric orbits, with drag-free operation, armlengths of some million $\mathrm{km}$ and heterodyne laser interferometers along the arms. "eLISA"/"NGO" is one specific incarnation of that concept under study in 2012.

\section{Conclusion}

Despite the drawbacks in 2011 and 2012, the scientific interest in LISA is stronger than ever before, the technology is well developed, the team is strong and convinced that LISA must fly in the early 2020s, and is committed to work hard to make that happen.

\section{References}

1. LISA: Unveiling a hidden universe. Technical report ESA/SRE(2011) 3, Noordwijk, Paris (2011). http://sci.esa.int/lisa/48364

2. Reich, E.S.: Europe makes do without NASA. Nature 471, 421 (2011). doi:10.1038/471421a

3. NGO: Revealing a hidden universe: opening a new chapter of discovery. Technical report ESA/SRE(2011) 19, Noordwijk, Paris (2011). http://sci.esa.int/ngo/49839 